Original scientific paper

\title{
Data Storage Devices in Science Fiction and Fantasy Movies
}

\author{
Domagoj Bebić \\ Faculty of Political Science, University of Zagreb \\ Lepušićeva 6, Zagreb, Croatia \\ Domagoj@edemokracija.hr
}

\begin{abstract}
Summary
Can we think about, make conclusions, observe, or predict social issues through movies? Through the analysis of selected science fiction and fantasy movies, the goal of this research is to identity the depiction of data storage technology and devices to see if the solutions offered were realistic, possible, and plausible or were just imaginable notions contrived for a movie script. For this purpose, several science fiction and fantasy movies are analyzed: 2001: A Space Odyssey (2001), Star Wars (1977), Superman (1978), Star Trek III: The Search for Spock (1984), Star Trek: Generations (1994), Johnny Mnemonic (1995), Minority Report (2002), and The Avengers (2012). The analysis showed each of the selected movies presented several revolutionary solutions for data storage devices. Several of the movies presented technology that is not yet available but probably will be in the near future. In detecting correlations between realistic technological developments and fantasy movie ideas, the analysis showed that while several movies accurately predicted some of the contemporary technological solutions for data storage, several just made fictional usage of these kinds of devices.
\end{abstract}

Key words: science fiction, fantasy, movies, data storage device, popular culture, technology

\section{Introduction}

In the pilot episode of the original TV series Star Trek, "The Cage" (1965), a race of technologically advanced humanoids called Talosians, after they have examined the databases of the USS Enterprise (Spock: "Tapes, micro-records, everything"), they concluded about humans: "Their method of storing records is crude and consumed much time."

As humans, we have a limited capacity for data storage and therefore remembering memories and experiences is not sufficient. That is why there is a constant need to save memory, to save data, and to invent procedures, devices, or solutions for its storage. One part of those options is for data to be transferred on different media to be remembered / archived as means of transferring knowledge. Whether it's for a family photo album, a computer program, or a 
Fortune 500 company's business-critical systems, data storage is a must-have for nearly everyone (Zetta, 2016).

Furthermore, data are important in our lives; it also serves as a significant inspiration in popular culture, especially in movies. Maybe the best illustration of data storage caption in movies is the legendary communicator, from the Star Trek TV series, which, as portrayed in the original series, is acknowledged as the first depicted mobile device (Wikipedia, 2017). This paper focuses on science fiction and fantasy movies in order to determine whether such movies are imaginative representations of reality or are only fictional manifestations of the movie's plot. Many authors were keen to explain the connectivity of technology and science fiction and fantasy films (Isaac Asimov, 1988; Bruce Sterling, 1988; Larry Niven, 1998; Barbara Hambly, 1982; Dan Simmons, 1989; Robert Heinlein, 1973). Also, several authors were involved in near future predictions throughout their science fiction work. In many cases, science fiction and fantasy movies are examples of genres that imply the free use of one's imagination and the depiction of currently unimaginable issues for contemporary culture and society. With this paper, however, the goal is to start identifying a number of segments within such movies that feature the use of data-storing devices, and decide whether these are presented as realistic solutions for data storage or just as imaginable solutions restricted to the movies. With a focus on detecting instances of stored data, transfer of data or back-up, this paper will explain such data devices in several science fiction and fantasy movies. The goal is to determine how science fiction and fantasy movies imagined and depicted data storage technology and whether these were realistic solutions or were only imaginary parts of popular culture. While this is the first notice, the movies for this paper are randomly selected due to their popularity and impact. For this purpose, several science fiction and fantasy movies will be analysed: 2001: A Space Odyssey (2001), Star Wars (1977), Superman (1978), Star Trek III: The Search for Spock (1984), Star Trek: Generations (1994), Johnny Mnemonic (1995), Minority Report (2002) and The Avengers (2012). Although there numerous technology-related films today, these are selected because of their $\mathrm{IMDb}^{1}$ rating and their popularity and impact. With this paper, the aim is not to explain the technology of the science fiction and fantasy movies themselves, but to provide review of data storage technology movie presentation in last forty years.

\footnotetext{
${ }^{1}$ The Internet Movie Database (abbreviated IMDb) is the world's most popular and authoritative source for movie, TV and celebrity content. The IMDb consumer site (www.imdb.com) is the \#1 movie website in the world with a combined web and mobile audience of more than 250 million unique monthly visitors (IMDb, 2017).
} 
D. Bebić, Data Storage Devices in Science Fiction and Fantasy Movies

\section{From punch cards to iCloud - An overview of data storage technology}

Mark Andrejevic, Alison Hearn, and Helen Kennedy (2015) declare that we are in the "age of big data" and that new analytical methods and businesses are emerging daily seeking to monetize this explosion of emerging data (Andrejevic et al., 2015: 379). Without technology and new, creative technological inventions, it would be impossible to capture, save, and store data. Therefore, technology plays an important, even crucial, role in the multiplying new possibilities for efficient data storage.

As Machles (1999) has pointed out, people are fascinated by the wonderful abilities of modern electronics and many have recorded countless hours of data on computers and other devices such as video recorders, both at home and at work. Furthermore, Machles (1999) explained that the same way of thinking has occurred with the use of compact discs and computer information. Documents that once were painstakingly handwritten or typed were replaced quickly by the use of computer discs, electronic copies, and similar storage media (Machles, 1999: 404). Today, we are witnessing the surge of modern, fast, and efficient data storage technology as every form of technological invention and data storage technology developed through the course of human history has been completely transformed. Today, the cloud is not just making data storage easier and more convenient-it is providing a platform for businesses and services, thereby building the next era of computing (Zetta, 2016).

The article (Gadgets, 2015) describes the chronological development of media for data storage from the eighteenth century up until the end of the twentieth century. As the authors explain in their article, the first technological data storage device consisted of punch cards, from 1725. After that, Alexander Bain, the inventor of the fax machine and the electric printing telegraph, patented punched tape in 1846. Selection tubes were invented in 1946 and magnetic tape in 1950. In the sixties, compact cassettes, the magnetic drum, and the floppy disc were invented. And in the seventies, the LaserDisc (1972) and the compact disc (1979) were invented. From the nineties, DVD and USB discs were significant data storage devices.

All these devices were important technological inventions but, with their development, also their storage improved. Today we are well acquainted with modern data storage media such as memory cards and USB discs but also cloudbased technology. Bryan Clark (2015) explains that hard drive technology is getting cheaper and storage capacity is improving and there is the move toward smaller internal storage is largely due to the expanding use of cloud-based technologies in order to store data, files, photos, videos, and more. 


\section{Movies as an important popular culture genre in the explanation of important social issues and concepts}

With the rise and popularization of media technologies, popular culture also has undergone a significant rise in popularity. Danesi (2015) defines popular culture as a "culture for the masses" and, because it is oriented toward people, it is one of the best representations of society's development (Danesi, 2015: 25). The possibilities of digital technology have contributed to a higher access to the genres and versions of popular culture to an ever-widening audience. Beer and Burrows (2013) assert that popular culture is at the center of the transformations that have facilitated the accumulation of digital data; but it is also at the heart of the issues and debates that face the social sciences (Beer, Burrows, 2013: 47). That is to say, we need to think about the way in which popular culture is folded into this "performativity of circulation" (Beer \& Burows, 2010: 50). As Laura Grindstaff (2008) has explained, probably the best-known strand of sociological research on popular culture is the "production of culture" perspective, which refers to the empirical study of culture-producing organizations within specific institutional contexts.

Today, while there are more and more versions and genres of popular culture, movies present an important and prominent part of popular culture. According to Jarvie (1978), at one time, movies were beneath the notice of academics and they were ranked lower than such acceptable forms of relaxation as detective mysteries (Jarvie, 1978: 374). Claudia A. Barriga, Michael A. Shapiro, and Marissa L. Fernandez (2010) explain that movies, particularly certain genres such as science fiction, medical dramas, and catastrophe films, often contain bits of scientific information with varying degrees of accuracy. There is evidence that the impact of fictional messages about unfamiliar groups may influence our beliefs about them just as much, or more, than nonfictional messages (Slater, 1990), showing that in certain cases fiction may have more influence than educational or informative messages. Anthony M. Hudock, Jr. and Sherry A. Gallagher Warden (2001) claim that several articles have discussed unique aspects of fictional movies, such as the richness and variety of content, emotive substance, and flexibility for use in assignments, making them appropriate for learning and training in the classroom (Chandler, 1997; Gladding, 1994; Gladstein \& Feldstein, 1983; Koch \& Dollarhide, 2000; Maynard, 1996; Voller \& Widdows, 1993). This indicates that movies play a significant role in the perception and imagination of future events, and they can provide solutions and ideas for technological and social issues of contemporary times. That is why this research seeks to examine the possibility of connections between the popular culture representation of realistic issues and needed solutions, in this case for data storage.

The role of movies on human perception and imagination is also explored by Aik-Ling Tan, Jennifer Ann Jocz, and Junqing Zhai (2017) in their analysis, Spiderman and Science: How Students' Perceptions of Scientists Are Shaped by 
Popular Media. In this research, the authors concluded that popular media, such as the Spiderman movies, have an influence on how young children perceive science and the work of scientists and that it is important to provide children with images and descriptions of a variety of science careers in order to motivate them to pursue further study in science and possibly science as a career.

Several authors (Erigha, 2016; Laan, 2010) also have wanted to identify the connections between movie presentations and realistic perceptions of common issues. While underlying themes of sci-fi media tackle contemporary, social, political, moral, religious, technological, and environmental issues (ChowWhite, Deveau, \& Adams, 2015), sci-fi filmmakers also make philosophical conjectures about the world, human existence, and the future (Erigha, 2016: 550). Andrew Milner and Sean Redmond (2015) consider science fiction to be a genre that challenges and critiques the status quo and has the ability to imagine other worlds that work in opposition and contradiction to the habitus of contemporary life (Milner, Redmond, 2015: 4).

In the context of technology presentation and science fiction movies one important name is Frank Herbert and his epic science fiction novel Dune (1965). Dune is considered as one of the greatest science fiction novels of all time (Dune novels, 2009). The first novel also inspired a 1984 film adaptation by David Lynch, the 2000 Sci-Fi Channel miniseries Frank Herbert's Dune and its 2003 sequel Frank Herbert's Children of Dune (which combines the events of Dune Messiah and Children of Dune), computer games, several board games, songs, and a series of followups, including prequels and sequels, that were cowritten by Kevin J. Anderson and the author's son, Brian Herbert, starting in 1999 (Wikipedia, 2017). On the other hand, many films are also involved in technological prediction of the future Iron Man $(2008$ - 2013), The Matrix trilogy (1999 - 2003), Her (2013), Eternal Sunshine of the Spotless Mind (2004), Total Recall (the 2012 remake), Elysium (2013), Prometheus (2012), Ender's Game (2013), Paprika (2006), Tron: Legacy (2010), Wall-E (2008) (Chung, 2014). This paper also contributes to the theoretical explanation of technological presentation of data storage.

\section{Imagination or reality: data storage technology in science fiction and fantasy movies}

As has been stated, the goal of this research is to examine a selection of science fiction and fantasy movies to identify data storage technology and devices and determine whether these movie data storage solutions were realistic presentations of possible and realistic data storage solutions or were just imaginable notions contrived for a movie script.

The analysis includes several science fiction and fantasy movies: 2001: A Space Odyssey (2001), Star Wars (1977), Superman (1978), Star Trek III: The Search for Spock (1984), Star Trek: Generations (1994), Johnny Mnemonic (1995), Minority Report (2002) and The Avengers (2012). These productions are pro- 
cessed chronologically in order to detect the evolution of technology and movie solutions for data storage. The goal of this analysis is not to discuss or analyse any particular narrative of a movie or story context, but to detect data storage technology and the movie's solution for its storage.

\section{1: A Space Odyssey (1968)}

In 1968, many of the technological solutions depicted in this movie were just ideas but are today realistic, and most of these ideas have come true and are part of contemporary technology. Fifty years ago this movie predicted technological innovations that have become possible in the last decade.

Among the interesting data from this movie is a scene of pilots watching news from video pads (BBC 12 World Tonight); today, of course, we can connect to numerous news sources with tablets or any touch screen. In addition, when it comes to writing down important space station measurements, the pilots are shown writing them down with a pen on a notepad. Another innovative technological solution from this movie is the role of the main computer. The computer-HAL 9000 - actually was the ultimate villain. In his final confrontation with the rebellious computer HAL 9000, astronaut Dr. David Bowman (aka Dave) has no other option but to remove HAL's memory from the spaceship's Logic Memory Center, which is portrayed as a small room containing hundreds of memory slots. In the movie, we can see how Dave is taking out the most important ones. Data memory is presented on numbered transparent PVC plates (separately for memory and for logic).

\section{Star Wars (1977)}

In the first movie of the Star Wars Space Saga (Star Wars IV: A New Hope) from 1977, the story is concretized around stolen Death Star plans, which are stored on a thin memory card that is hidden in the R2D2 Unit (or just R2). In the movie, this memory card is depicted as a data storage device while a robot (R2) is shown as displaying or projecting the data in the form of a hologram. The memory card presented in the movie Star Wars is a combination of the floppy disc that was available at the time and the compact disc (CD) that was invented only two years after the movie's appearance. Also, at the time the movie was recorded, no technology existed that could present data in the way that is shown in the movie. Today we are using similar technology with CDs, memory cards, or USB discs and projectors that can present data. The movie also predicts a 3D presentation of the data. And while the movie is revolutionary in its explanation and presentation of the universe and distant worlds, it is equally revolutionary in the technological solutions it depicts for data storage devices. Today, 3D presentation technology is still in development, and we can say that this movie predicted this kind of technological data presentation. 


\section{Superman (1978)}

The crystal in Superman (1978) presents an out-Earth technology that not only contains data, but also acts as a power source that is not known to the human race. Jor-El from the planet Krypton sends this crystal in a spaceship with his son Kal-El to the Earth before the destruction of his planet. Not only does the crystal contain all the recorded data from the destroyed Krypton, it also possesses an indescribable power. Besides data, feelings, and power, the crystal also has the ability to present data: when it is placed on a portal, the crystal projects a hologrammatic message.

This is the text spoken by Jor-El to his son in the 1978 Superman movie:

"The total accumulation of all knowledge spanning the twenty-eight known galaxies is embedded in the crystals which I have sent along with you. Study them well, my son. Learn from them. "

In Superman, the crystal is presented as a technology that is not available for humans and humans are unable to use it. That is why this technology for data storage is still not available for humans.

\section{Star Trek III: The Search for Spock (1984) and Star Trek: Generations (1994)}

From the beginning of the Star Trek movie series (1979), we can see extensive usage of the tablets that are called control pads. In the very first movie when Captain Kirk is transported to the Enterprise and its crew is rushing to make the Enterprise functional in a short time due to an unexpected mission to stop an alien force hidden in a cloud of energy moving toward Earth, we can see that most of the crew are using control pads to perform their work assignment.

In The Search for Spock, an original series movie (1984), when Klingon Commander Kruge is contacted by Valkris who wants to transmit data about Project Genesis, she does so via porting with a kind of cassette device. At the time the movie was made, cassette technology was available and familiar, the same technology we can find on a Klingon ship, which leads us to conclude that this is widespread intergalactic technology. In the Next Generations Star Trek series movie, Star Trek: Generations (1994), we can notice that after Captain Picard receives the information that his brother Robert and his nephew Rene have died in a fire back on Earth, he invokes memories of his family by looking at an oldfashioned photo album. In the scene, the data are saved in an old-fashioned way and there is not much technology involved other than the lighting frames of the photo. However, while in this scene there was no technological data storage, in other scenes there were a few revolutionary data storage solutions. For example, the multidimensional Data Room called Stellar Cartography where Captain Picard and Commander Data are trying to understand the destination of Doctor Soran. Stellar Cartography is not a holodeck; rather, it is an interactive multimedia multiscreen database. This technology is later elaborated in other movies, 
for example, the X-Men movies, but still is not among contemporary technological possibilities.

Also, another interesting scene occurs when Doctor Soran pays a Klingon to get him safely to the planet of Veridian III; he holds out a designed memory stick, saying: "This contains all the information you'll need to make a trilithium weapon." He emphasizes that the stick is coded so he can get safely to the planet surface of Veridian III, after which he will send them the decryption sequence.

\section{Johnny Mnemonic (1995)}

The plot of this movie from 1995 takes place in 2021. In a world of corporations, which control information and access to it, the safest data transistor is the human brain, which, with implants, can gain significant capacity. While the human mind has limited memory and capacity and is not a safe data transistor, in this movie such an enhanced brain is presented as the only safe solution for data storage. The main character, Johnny Mnemonic, has a brain capacity of 80 GB, but with hardware addition (memory doubler) the capacity is increased to $160 \mathrm{~GB}$. A main plot driver of the movie falls into place when Johnny decides to upload $320 \mathrm{~GB}$ into his mind, which overloads his present mind. The data held within such an enhanced mind is protected with a picture password taken from random TV pictures, which are selected during the data transmission with a specially designed remote control. These photos can be printed, scanned, and faxed. Therefore, while we are dealing with technology that is still not yet available, this technology is transmitted over media channels available at the time (telephone, scanner, fax machine, etc.). In this movie, there is an interesting demonstration of cyberspace. When the main character, Johnny, connects to cyberspace via a dial-up, the cyberspace realm is presented through two-dimensional animation as a three-dimensional world.

In addition, another interesting data storage solution we can identify in this movie concerns documents, especially passports. A passport is presented as a data-card, which at border control is put in a special reader. The passport is designed as a regular document, but its usage is different. Today, a document and a passport scanner are similar technology.

\section{Minority Report (2002)}

The plot of the movie Minority Report (2002) takes place in 2054. Most of the movie's dynamics take place inside the Precrime Unit where visions of future crime are pre-seen by Precognitive persons (Precogs) inside a biopool. These crime visions are stored on a separate interface on plexy plates in the form of multidimensional images. These plates are then inserted in different portals dedicated to data manipulation and additional information and data search, which include the detective's activities, such as defining the surroundings (area and address) where a certain crime is going to take place. To perform these ac- 
tions, the detective manipulates the video information on a hologrammatic interface using special data gloves.

The memories that form a life can also be stored on smaller glass boards in the form of a video. The data from these boards are projected as a hologram video.

\section{The Avengers (2012)}

The Avengers (2012) deals with the realization of the concept of S.H.I.E.L.D. (Strategic Hazard Intervention Espionage Logistics Directorate), which was mentioned in the movie Iron Man 3. In the scene where agent XX meets with Tony Stark, he gives him a special kind of two-sided laptop as a device for viewing secret data. Another interesting idea is realized in this movie scene and deals with data presentation. On the laptop, there are multidimensional data and folders, which are opened on several hologrammatic displays. We can see that these data are interactive because in one moment, Tony Stark can not only see, but also can accept the hologram's data. While this technology is not yet available today, we can witness the development of 3D printing and highly interactive devices (computers, mobile phones, etc.) that parallel this development.

Another interesting technological solution in this movie is the possibility of easy data transmission. In the movie, data can be transmitted from one computer to another with just a slide of the finger. This presents one possible solution for faster data transmission in the future.

\section{Conclusion}

While movies present a number of important genres of popular culture, the aim of this paper was to determine whether science fiction and fantasy movies in particular could predict technological solutions for future issues. In this paper main focus was detecting presentation of data storage technology in popular science fiction and fantasy films in last 40 years. For this purpose several science fiction and fantasy movies were randomly selected for the initial exploration of the link between data storage technology, film and projection of the future. By analysing several science fiction and fantasy movies-2001: A Space Odyssey (2001), Star Wars (1977), Superman (1978), Star Trek III: The Search for Spock (1984), Star Trek: Generations (1994), Johnny Mnemonic (1995), Minority Report (2002), and The Avengers (2012) the goal was to identify and explain how data storage devices were presented in fantasy and science fiction movies. In order to determine if these movie data storage solutions were realistic presentations of possible and realistic data storage solutions or just imaginable parts of a movie script. As shown by the analysis, each of the selected movies presented several revolutionary solutions for data storage devices. The initial analysis revealed that movies like 2001: A Space Odyssey (2001), Star Wars (1977), Superman (1978), and Star Trek III (1984) deal with technological devices for data storage, while, for example, more recent movies like Johnny Mnemonic (1995) use the human brain as a device for data transmission and 
storage, while Minority Report (2002) uses human memories as a form of data that can be saved on a technological device. Several movies presented technology that wasn't available at the time, but was invented later on. For example, 2001: A Space Odyssey (1968) provided a large amount of innovative technological solutions like virtual (video) border control, voice ID (voice print identification), traveling in zero gravity, and video conversations (picture phone), tablets - all of which are inventions of contemporary time. Several movies presented technology that is still not yet available but probably will be in the near future. These include, for example, the hologrammatic messages in Star Wars (1977) and Superman (1978), or 3D demonstration of the data and easy data transmission throughout several platforms in The Avengers (2012). Interesting data were included in Star Trek III (1984) and Star Trek: Generations (1994). While Star Trek: Generations (1994) movie presented the first version of a USB device, Star Trek III (1984) had several traditional technological procedures for data storage, like video cassettes and photo albums. As regards any correlation between realistic technological developments and fantasy movie ideas, the analysis showed that while several movies really predicted some of the contemporary technological solutions for data storage, several made merely fictional usage of these kinds of devices. For example in Superman (1978), the data storage device was presented in the form of a crystal, but also it contained indescribable power that was both magical and unrealistic. In addition, Johnny Mnemonic (1995) presented the human brain as an upgraded device and in Minority Report (2002), memories are used as accessed and transferred data. The interesting data is that the newer the films is, the data storage technology is becoming smaller, more abstractedly and more interrogated with the human. For example, in Johnny Mnemonic (1995) and in in Minority Report (2002) we can see that data storage is connected with a human, part of his DNA. While in The Avengers (2012), we have interactive technological solutions for data storage. In contrast to older movies (Star Trek, Star Wars, etc.) which presented data storage technology as devices, in movies that are more recent we have projection of imaginary space for data storage. This paper presents initial idea for further empirical and theoretical research. For further research, the goal is to expand the sample and include more science fiction and fantasy films and to conduct a qualitative and quantitative data study. By this approach, it would be possible to provide empirical data for this concept and to make more solid conclusions about the predictions in the future. With this analysis, it is possible to detect that more recent science fiction and fantasy films project data storage devices as invisible cyber space, similar to cloud computing technology that is present today and will probably be more developed in the future. Steven Hrotic (2014) asserts that science fiction will do as it always has done, create new purposes, not for the purpose of bringing about or preparing us for a technological future, but to teach us to live in the technological present. In that context, mov- 
ies are still a part of popular culture, but they are also representations of the human imagination and ideas.

In the context of the issues and further developments in the future (not only for data storage), one can conclude with the Fleetwood Mac song "Don't Stop":

\section{"Don't stop thinking about tomorrow, Don't stop it'll soon be here, It'll be, better than before, Yesterday's gone, yesterday's gone"}

(Fleetwood Mac, 1968)

\section{References}

Andrejevic, Mark; Hearn, Alison; Kennedy, Helen. Cultural studies of data mining: Introduction // European Journal of Cultural Studies. 18(2015), 4-5; 379-394

Barriga, Claudia; Shapiro, Michael; Fernandez, Marissa. Science Information in Fictional Movies: Effects of Context and Gender // Science Communication. 32(2010), 1; 3-24

Beer, David; Burrows, Roger. (2013). Popular Culture, Digital Archives and the New Social Life of Data // Theory, Culture \& Society. 30(2013) 4; 47-71

Bryan Clark. From Punch Cards to Holograms - A Short History of Data Storage. (2015) http://www.makeuseof.com/tag/punch-cards-holograms-short-history-data-storage/, (Access date 1 July, 2017);

Chandler, Theresa. An alternative comprehensive final exam: The integrated paper // Teaching Sociology / Hudock, N1; Gallagher Warden, N2. (ed). Youngstown: The Family Journal, 2001, 3

Chung, Becky. 13 Movies That Explore The Future Of Technology. (2014). https://creators.vice. com/en_au/article/53wgp5/13-movies-that-explore-the-future-of-technology (Access date 12 October, 2017)

Communicator (Star Trek). (2017). https://en.wikipedia.org/wiki/Communicator_\%28Star_Trek\%29, (Access date 1 July, 2017)

Danesi, Marcel. Popular Culture: Introductory Perspectives: 3rd Edition. London: The Rowman and Littlefield Publishing Group, 2015

Dune (novel). (2017) https://en.wikipedia.org/wiki/Dune_(novel) (Access date 12 October, 2017)

Dune novels. (2009) http://www.dunenovels.com/ (Access date 12 October, 2017)

Erigha, Maryann. Do African Americans Direct Science Fiction or Blockbuster Franchise Movies? Race, Genre, and Contemporary Hollywood // Journal of Black Studies, 47(2016) 6; 550569

Gadgets Fosfor. History of data storage. (2015). http://gadgets.fosfor.se/history-of-data-storage/. (Access date 28 June, 2017);

Gladding, Samuel T. Teaching family counseling through the use of fiction // Counselor Education and Supervision, 33(1994) 3; 191-200

Gladstein, Gerald A.; JoAnn C. Feldstein. Using film to increase counselor empathic experiences // Counselor education and supervision 23(1983)2; 125-131

Grindstaff, Laura. Culture and popular culture: A case for sociology // The ANNALS of the American Academy of Political and Social Science, 619(2008)1; 206-222

Hambly, Barbara. Time of the Dark. Integrated Media: New York, 1982.

Heinlein, Robert. Time Enough for Love. Ace Books: New York, 1973.

History of Data Storage Technology. (2016). http://www.zetta.net/about/blog/history-datastorage-technology, (Access date 28 June, 2017)

Hudock, Anthony; Gallagher Warden. Using Movies to Teach Family Systems Concepts // The Family Journal: Counseling and Therapy For Couples And Families, 9(2001)2; 116-121 
IMDb. (2017) http://www.imdb.com/pressroom/?ref =ft pr (Access date 12 October, 2017)

Jarvie, Ian, Charles. Seeing through Movies // Phil.Soc.Sci, 8(1978); 374-397

Koch, Gary, and Colette T. Dollarhide. Using a popular film in counselor education: Good Will Hunting as a teaching tool // Counselor Education and Supervision 39(2000)3; 203-210

Machles, David. Electronic Media Storage // AAOHN Journal 47, 10(1999)10; 494-498

Maynard, Peter E. Teaching family therapy theory: Do something different // American Journal of Family Therapy, 24(1996)3; 195-205

Milner, Andrew. Sean Redmond. Introduction to the special issue on science fiction // Thesis Eleven, 131(2015); 3-11

Niven, Larry; Anderson, Poul; Ing, Dean. Man-Kzin Wars. Baen Publishing Enterprises: New York, 1998.

Simmons, Dan. Hyperion Cantos. Doubleday: New York, 1989.

Sterling, Bruce. Islands in the Net. New York: Ace Books, 1988.

Tan, Aik-Ling, Jennifer Ann Jocz, and Junqing Zhai. Spiderman and science: How students' perceptions of scientists are shaped by popular media // Public Understanding of Science 26(2017)5; 520-530

Van der Laan, J. M. Frankenstein as science fiction and fact // Bulletin of Science, Technology \& Society, 30(2010)4; 298-304

Voller, Peter, and Steven Widdows. Feature films as text: A framework for classroom use // ELT Journal, 47(1993)4; 342-353 